\title{
Cashless Japan: Unlocking Influential Risk on Mobile Payment Service
}

\author{
Wei-Lun Chang ${ }^{1}$ (D) $\cdot$ Li-Ming Chen $^{2} \cdot$ Takako Hashimoto $^{3}$ \\ Accepted: 15 June 2021 / Published online: 25 June 2021 \\ (C) The Author(s), under exclusive licence to Springer Science+Business Media, LLC, part of Springer Nature 2021
}

\begin{abstract}
In Japan, cashless is not yet popular but government and companies are devoted to the development of mobile payment methods. This research collected 241 Japanese users and applied decision trees algorithm. Six types of perceived risks (financial, privacy, performance, psychological, security, and time) were used and the categorized class is intention to use mobile payment (low, medium, and high). We also compared different competitive models to examine the performance, including decision trees, $\mathrm{kNN}$, Naïve Bayes, SVM, and logistic regression and decision trees outperformed among all models. The findings indicated that privacy and performance risks are import to Japanese users. Safe, secured, reliable, and fast mobile payment environment are more important to low intention users (less concerns about financial risk). Financial loss, safe, secured, reliable, and fast mobile payment environment are more important to medium intention users (less concerns about time and security risk). Monetary loss, safe, reliable, and fast mobile payment environment are more important to high intention users (less concerns about security risk and psychological risk). The results can help Japanese companies unlock the perceived risk on mobile payment and furnish appropriate strategies to improve usage.
\end{abstract}

Keywords Mobile payment $\cdot$ Perceived risk $\cdot$ Decision trees $\cdot$ Cashless

\section{Introduction}

The global mobile payment market exceeds $\$ 601.3$ billion (Dighe, 2018) and is expected to reach $\$ 4573.8$ billion by 2023. Other sources (MarketsandMarkets, 2018) predict that the global digital payment market will be worth $\$ 10.07$ trillion by 2026, with $5 \mathrm{G}$ being the key to the development. The Asia Pacific, is predicted to generate a revenue of $\$ 3.62$ trillion in the year 2026, owing to its extensive market penetration towards digital payment coupled with cashless

\section{Wei-Lun Chang \\ wlchang@ntut.edu.tw \\ Li-Ming Chen \\ lmchen@nccu.edu.tw \\ Takako Hashimoto \\ takako@cuc.ac.jp}

1 Department of Business Management, National Taipei University of Technology, 1, Sec. 3, Zhongxiao E. Rd, Taipei 10608, Taiwan

2 Department of Business Administration, National Chengchi University, NO64,Sec.2,ZhiNan Rd.,Wenshan District, Taipei City 11605, Taiwan

3 Commerce and Economics, Chiba University of Commerce, 1-3-1 Konodai, Chiba 272-8512, Japan economy. The more developed markets seeking to become the $5 \mathrm{G}$ global leaders (e.g. Japan), are expected to see rapid $5 \mathrm{G}$ growth by 2025 , accounting for half of total mobile connections. However, 5G opportunities are less attractive because there is still more potential for leveraging the capabilities of 4G. The focus across the Asia Pacific markets is to push innovation in areas such as mobile commerce and payment (GSM Association, 2019).

An analysis of the popularity of mobile payment across Asia, including China (Alipay), India (Visa), and the US (Apple Pay and Android Pay), undertaken by Mordor Intelligence (2018), uncovered that threats such as cybercrime form the major barriers to mobile payment adoption. Particularly, credit cards are still the top payment in Japan, following by cash over the counter. PayPay, a mobile payment application is rapidly gaining popularity since 2018 in Japan according to Asia Pacific eCommerce and Payments Guide (2020). Payment method changes have also contributed to the transition from cash to cashless payment in retail (Arvidsson, 2014). Further, payments via smartphones have raised concerns about risks amongst consumers (Saridakis et al., 2016). Existing literature showed that favorable attitude (Park et al., 2019) and service quality (Liébana-Cabanillas et al., 2019) positively influenced consumer willingness to use mobile payment. Security (Oliveira et al., 2016; Shao et al., 2019), trust (Zhou, 2013), and risk (Cocosila \& 
Trabelsi, 2016) were identified as the most affecting factors on mobile payment adoption. Although the mobile payment industry has arguably reached maturity, concerns regarding privacy and security risks persist (Albashrawi \& Motiwalla, 2019).

Existing studies have investigated factors that negatively affect perceived risks in mobile banking or online shopping environment (e.g. Kim \& Lennon, 2013; Mann \& Sahni, 2013), as these perceived risks slow down mobile payment industry development (Choi \& Choi, 2017). They also negatively influence mobile payment consumer acceptance (Yang et al., 2015) and trust in this payment method (Park et al., 2019). In our study, we categories intention to use mobile payment from low, medium, to high across the users in Japan. Following Dey (2002), Kabari and Nwachukwu (2013), and Ramezankhani et al. (2016), this research uses a decision tree classification method to investigate the link between perceived risks of mobile payment based on (Yang et al., 2015; Thakur \& Srivastava, 2014) and the three categories (intention to use). The study expects to answer the calls by Fahey (2019) and findings from the Mobile Economy Report on GSMA (2019) and aims to provide insights into the barriers to mobile payment adoption in the developed Asia Pacific, particularly as Japan is set to lead the $5 \mathrm{G}$ innovation. Hence, the following research questions are put forth:

\section{RQ1: What are the critical risks for different categories of mobile payment users? \\ RQ2: What are the differences among the categories of mobile payment users?}

\section{Related Literature}

\subsection{Mobile Payment}

The process of payment in which mobile devices are used to execute transactions for products or services, anytime and anywhere, is known as mobile payment. It is an alternative to traditional payment methods by cash or credit card and uses communication devices (Dahlberg et al., 2008) to carry out the payment authorization and execution of financial transactions (De et al., 2018). Mobile payments have also been shown to increase sales in physical stores (Liu et al., 2015). The acceptance of mobile payment depends on the willingness to accept new technologies (Liébana-Cabanillas and Lara-Rubio, 2017; Qasim \& Abu-Shanab, 2016). New smartphone functions, such as near field communication, support mobile payment use (Oliveira et al., 2016), reducing costs and increasing retail outlet profitability (Chen \& Li, 2017). Previous experiences (individual factor) and market competition (external factor) also influence the willingness to adopt mobile payment (Zhu et al., 2017). Studies in the literature converge on the factors affecting mobile payment adoption: acceptance, risk, perception, trust, and willingness (Liébana-Cabanillas et al., 2019; Cocosila \& Trabelsi, 2016; Zhou, 2013; Park et al., 2019; De et al., 2018; Oliveira et al., 2016; Shao et al., 2019); these factors are used in this study. Regarding the relation between acceptance and mobile payment use, mobile payment enhances user experience and offers competitive differentiation in the market (Hayashi \& Bradford, 2014). Also, smartphone limitations negatively influence the user experience, which varies widely due to the variety of smartphone models and application vendors (Zhou, 2014). Additionally, the connection between mobile payment and traditional payment methods plays an important role in building trust in mobile payment (Cao et al., 2018).

Cybersecurity issues tend to dominate the decision to adopt mobile payment. Perceived threats of hackers and malware that threatens mobile devices have been shown to be the major reasons for their low usage rate (Zhou, 2014). Mobile payments may reduce financial losses in retail outlets, as they have been proven to be safer than credit card payments (Hayashi \& Bradford, 2014). Yet early adoption of mobile payment has created perceptions of risks and uncertainty (Xin et al., 2015), in particular amongst consumers who have lower trust in technologies and mobile services driven by concerns related with privacy, security, and erroneous payment transactions (Dinh et al., 2018). With regard to the perception factor, studies show (Xin et al., 2015) that consumers are uncertain and do not trust mobile payment companies. Additionally, self-efficacy is a major factor influencing the resolution to use mobile payment in stores ( $\mathrm{Nel} \&$ Heyns, 2017). Thus, when more retailers make mobile payment available to consumers this will lead to the improvement of user perceptions and self-efficacy (Dinh et al., 2018). Research shows that once consumers form trust towards mobile payment they continue to use this method according to their understanding of the level of security (Zhu et al., 2017). Trust in mobile payment providers is a significant factor affecting willingness to use (Xin et al., 2015). Conversely, the availability of additional mobile services has little effect on use (Nel \& Heyns, 2017), as consumers may stop using mobile payment even if providers offered additional services (Zhou, 2014).

\subsection{Perceived Risks in Mobile Payment}

Perceived risk strongly influences the consumer decision process in any purchasing environment (Gillett, 1976). Perceived risk is defined as a likelihood of loss due to uncertainty related to unexpected outcomes when making purchase decisions (Featherman \& Pavlou, 2003). The ability to accept perceived risk affects financial transaction decisions (Forsythe \& Shi, 2003), as perceived risk has been linked to consumers' subjective expectations, thereby extending its influence on the 
mobile payment decision-making process. The literature on perceived risks has focused on the key risk categories: financial, privacy, performance, psychological, time, and security. Financial risk indicates a possible monetary loss due to the use of mobile payment methods (Featherman \& Pavlou, 2003). It is advantageous to use mobile payment when other payment methods incur higher costs (Luarn \& Lin, 2005) or when the cost of continuous usage leads to a possible financial loss. Additionally, financial risk is associated with monetary expenses and maintenance costs. The uncertainty regarding mobile payment authorization might increase mobile users' concerns (Yang et al., 2015), as system malfunction during financial transactions could lead to potential losses (Baganzi \& Lau, 2017).

Privacy risk indicates the risk of personal information exposure (Featherman \& Pavlou, 2003), as consumers are concerned about the exposure and misuse of their personal data involved in mobile payments. Disclosure and misuse of personal information cause consumers to lose control over their personal data (Khalilzadeh et al., 2017) allowing providers to harvest, process, transfer, and sell their personal information (Yang et al., 2015), thus helping these providers to gain insight on users' non-public data and shopping behavior (Thakur \& Srivastava, 2014). Sensitive information such as personal identification, credit card information, and other financial data makes many customers uncertain and concerned about their privacy (Baganzi \& Lau, 2017). Performance risk relates to system malfunctions that affect mobile payment services provided to users (Featherman \& Pavlou, 2003). Performance can be volatile due to the limitations in smartphone capabilities; this volatility in turn raises users' concerns (Yang et al., 2015). Consumers expect mobile payment to improve the efficiency of daily tasks (Khalilzadeh et al., 2017); however, the instability of wireless connections and the limited processing capabilities of mobile devices increase performance risk (Choi \& Choi, 2017).

Psychological risk refers to frustration, perceived anxiety, and psychological pressure (Lim, 2003). Compared to online payment or credit card payment, mobile payment is a novel and complex service. For example, consumers might feel anxious because of a failed transaction (Yang et al., 2015). Psychological risk of mobile payment is also associated with unfamiliarity, unreliability, and fear (Trachuk and Linder, 2017). Time risk indicates the delays experienced by using mobile payment because of user uncertainty, the learning curve of mobile applications, or the risk of an incomplete payment process (Featherman \& Pavlou, 2003). Moreover, consumers occasionally experience longer transaction time, causing inconvenience. The need for additional time to become experienced with the mobile payment system and to troubleshoot its problems is also a time risk factor affecting users (Choi \& Choi, 2017).
Finally, security risk refers to the risk of uncontrolled transactions and loss of financial information. It is also associated with the perceived payment method security, security of information at rest and in transit, and cybersecurity overall (Kolsaker \& Payne, 2002). Cybersecurity is the link between the perceived risk and the consumer attitude (Khalilzadeh et al., 2017); it assures information confidentiality, integrity, and service availability (Flavián et al., 2006). Consequently, sales increase only when the perceived security of the payment transaction data and other sensitive information is high (Thakur \& Srivastava, 2014).

\subsection{Mobile Payment in Japan}

The Ministry of Economy, Trade and Industry (METI) of Japan published the "Cashless Vision" in 2018 to promote cashless payments and declared the goal of achieving a $40 \%$ cashless payment ratio. The Cashless Promotion Council was established as an industry-academiagovernment collaboration to promote the Cashless Payments (Payments Japan, 2020). The Payments Japan conducted a quantitative and qualitative analysis of users to consider measures for the spread of cashless payment (Payments Japan Consumer and Business Insight Survey, 2020). The analysis addressed the reason that Japanese don't use mobile payments by a web-based survey with 5000 people in September 24 to 29 of 2019 . The results reported that only $19 \%$ of people carry a mobile payment method on a daily basis, and only $13 \%$ of people (about 700 people) use mobile payment at least once a month. Despite the fact that more than $80 \%$ of people are aware of mobile payments (e.g., Apple Pay, and Google Pay), $24 \%$ have contactless IC payment apps (e.g. Apple Pay, and Google Pay) installed on their mobile devices and only $14 \%$ usage. For code-based payment apps (e.g. Line Pay), $42 \%$ have them installed but only about $30 \%$ usage. As of 2019 , mobile payment methods had not been shown to be sufficiently widespread in Japan. Through a qualitative survey conducted by interviews with 15 more people, people do not use cashless payment because of lack of interest and need, concerns about overspending, lack of acceptance from the consumer's point of view, avoidance of complexity in household management, and convenience comparisons. These factors are considered to be largely related to the perceived risks classified in the existing literatures (Yang et al., 2015; Thakur \& Srivastava, 2014). The low popularity of cashless utilization is also illustrated in a survey report by the Ministry of Economy, Trade and Industry (METI) of Japan, which reported that Japan's cashless ratio was only about $20 \%$ in 2016 , which is significantly lower than that of other major countries (4060\%) (METI report, 2020). 


\section{Research Method}

\subsection{The Proposed Framework}

Figure 1 shows the conceptual model that enfolds a C4.5 decision tree learning algorithm (Quinlan, 1993). This study utilizes perceived risks as the attributes and degree of intention to use as the categories (i.e., low, medium, and high) in the decision trees algorithm. Existing literature classified perceived risks into distinct categories such as financial, privacy, performance, psychological, monetary, time, and security (Yang et al., 2015; Thakur \& Srivastava, 2014). We finally merged monetary risk with financial risk to adopt the six fundamental risks in mobile payment.

\subsection{C4.5 Decision Trees}

Decision trees as flowchart-like structures have been used for processing classification problems ever since the seminal work from Breiman et al. (1984). The basis for the classification process must be known prior to establishing the classification model. Additionally, tree-structured models are established based on class labels and using actual data entries to build up a concise model (Agrawal et al., 1998). According to these models, common characteristics and rules can be summarized and used to predict other unclassified or new data. Moreover, processes of decision trees include data training and testing processes. Each data entry in the training data

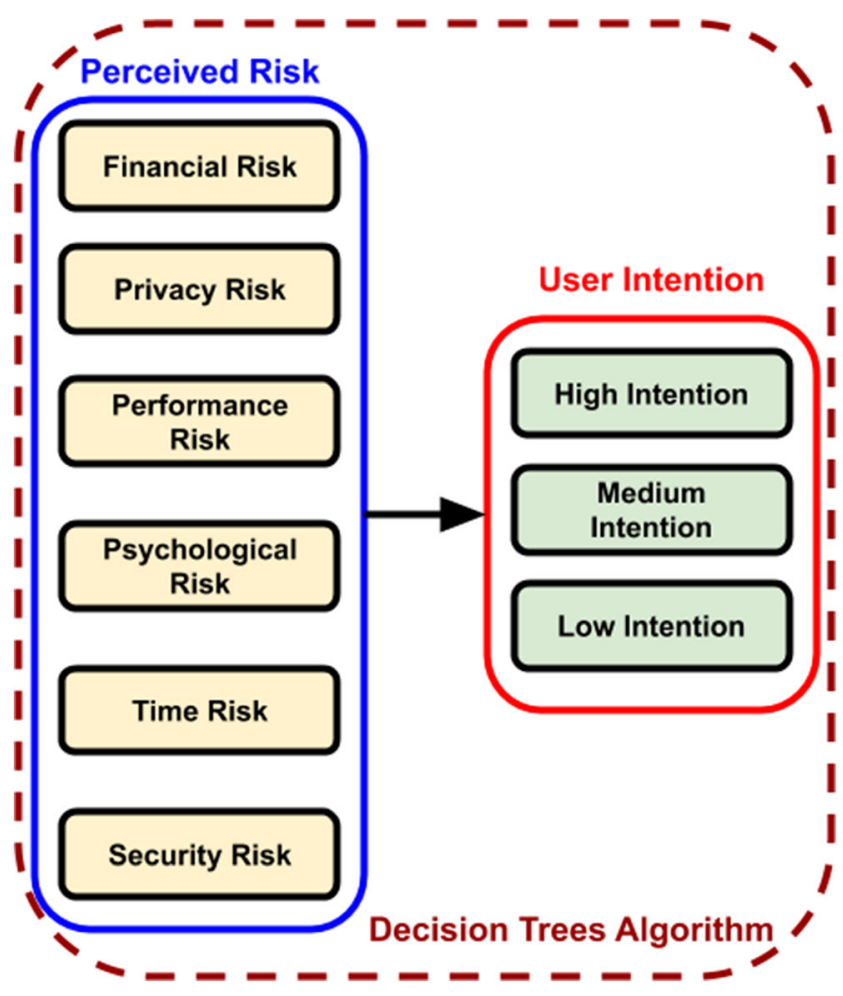

Fig. 1 The proposed framework is used to shape a decision tree based on data attributes. Each internal node represents a decision point and a testing condition, whereas each branch represents the testing results and the leaf nodes show the classification results. Finally, after defining a decision tree, its validity is verified by testing data. Figure 2 shows an example of a decision tree, which includes four nodal types. The first type is the root node (starting node), where the processing of new data begins. The second type is the child node (i.e., internal node), which represents individual testing conditions and stores minimal data to determine the subsequent data branch. Acting as a link between these two nodes is the nodal bridge. Each branch represents a testing result and functions as a nodal bridge. The fourth type is the leaf node, which represents various class labels. All data landing on this node exhibits identical characteristics.

This study applies a C4.5 algorithm that uses information entropy to build a decision tree based on training data. Each node of the decision tree represents an attribute of the data that can effectively split samples into subsets of class or other attributes. The calculation of the $\mathrm{C} 4.5$ algorithm can be divided into Eq. (1) and Eq. (2). In Eq. (1), $D$ is the data set that includes $m$ (classified results), where the probability of each result $\mathrm{m}$ is $p_{m}$. The $\mathrm{C} 4.5$ uses a gain ratio to solve this problem by considering splitting information. For example, if we have a feature $\mathrm{D}$ that has a distinct value for each record, then $\operatorname{Info}(D)$ is 0, thus Gain(A) is maximal. In Eq. (2), GainRatio(A)is the proportion of information generated by the split that is useful for the classification. This study uses the notion of GainRatio to rank attributes and to build decision trees. Hence, each node is located with the attribute with highest GainRatio among the attributes (not yet considered) in the path from the root.

$$
\begin{aligned}
\operatorname{Gain}(A) & =\operatorname{Info}(D)-\operatorname{Info}_{A}(D), \text { where } \operatorname{Info}(D) \\
& =\sum_{i=1}^{m}-p_{i} \log _{2} p_{i}
\end{aligned}
$$

Fig. 2 An algorithm of C4.5 decision trees 


$$
\begin{aligned}
\operatorname{SplitInfo}_{A}(D)= & -\sum_{j=1}^{v} \frac{\left|D_{j}\right|}{|D|} \\
& \times \log _{2} \frac{\left|D_{j}\right|}{|D|} \text { and GainRatio }(A) \\
= & \frac{\operatorname{Gain}(A)}{\operatorname{SplitInfo}_{A}(D)}
\end{aligned}
$$

\subsection{Naïve Bayes Classifier}

Naïve Bayes Classifier is a simple method through statistics theory. Furthermore, it has been widely used in classification problems because it is a fundamental technique in machine learning. According to Han et al. (2012), definition of Naïve Bayes is a probabilistic classifier based on Bayes' theorem (Eq. (3)).

$P(H \mid X)=\frac{P(X \mid H) \times P(H)}{P(X)}$

Bayesian classifiers have also exhibited high accuracy and speed when applied to large database. Suppose that there are m classes, $C_{1}, C_{2}, \ldots, C_{m}$. Given a tuple, $\mathrm{X}$, the classifier will predict that $\mathrm{X}$ belongs to the class having the highest posterior probability, conditioned on X. That is, the Naïve Bayesian classifier predicts that $\mathrm{X}$ belongs to the class $C_{i}$ if and only if

$P\left(C_{i} \mid X\right)>P\left(C_{j} \mid X\right)$ for $1 \leq \mathrm{j} \leq \mathrm{m}, \mathrm{j} \neq \mathrm{i}$

Thus, we maximize $\mathrm{P}\left(C_{i} \mid \mathrm{X}\right)$. The class $C_{i}$ for which $\mathrm{P}\left(C_{i} \mid\right.$ $\mathrm{X})$ is maximized is called the maximum posteriori hypothesis. By Bayes' theorem (Eq. 3.1),

$P\left(C_{i} \mid X\right)=\frac{P\left(X \mid C_{i}\right) \times P\left(C_{i}\right)}{P(X)}$

In other words, we predict that class label is the class $C_{i}$ when we calculate $\mathrm{P}\left(\mathrm{X} \mid C_{i}\right) \times \mathrm{P}\left(C_{i}\right)$ is the maximum.(Eq. (4)) By calculating the maximum of $\mathrm{P}\left(\mathrm{X} \mid C_{i}\right) \times \mathrm{P}\left(C_{i}\right)$,we obtain the highest probability of $\mathrm{X}$ belongs to class $C_{i}$, and we classify $\mathrm{X}$ in $C_{i}$.

\subsection{Support Vector Machine}

Support Vector Machine is a method for the classification of both linear and nonlinear data. It uses a nonlinear mapping to transform the original training data into a higher dimension. Within this new dimension, it searches for the linear optimal separating hyperplane. According to Han et al. (2012), although the training time of even the fastest SVMs can be extremely slow, they are highly accurate, owing to their ability to model complex nonlinear decision boundaries. They are much less prone to overfitting than other methods.

The SVM searches for the hyperplane with the largest margin; that is, the maximum marginal hyperplane(MMH). The associated margin gives the largest separation between classes. First, we take a simpler case for example. Let the dataset $\mathrm{D}$ be given as $\left(x_{1}, y_{1}\right),\left(x_{2}, y_{2}\right), \ldots,\left(x_{|\mathrm{D}|},\left(y_{|\mathrm{D}|}\right)\right.$, where $x_{i}$ is the set of training tuples with associated class labels, $y_{i}$. Each $y_{i}$ can take one of two values, either +1 or -1 (i.e., $y_{i} \in\{+1,-1\}$ ), corresponding to the classes buys_computer $=$ yes and buys_computer $=$ no, respectively. Therefore, two possible separating hyperplanes and their associated margins. We search for the largest margin hyperplane to approach the problem. A separating hyperplane can be written as $W \cdot X+b=0$, where $\mathrm{W}$ is a weight vector, namely, $\mathrm{W}=\left\{w_{1}, w_{2}, \ldots, w_{n}\right\} ; \mathrm{n}$ is the number of attributes; and $\mathrm{b}$ is a scalar, often referred to as a bias. If $\mathrm{X}=\left(x_{1}, x_{2}\right)$, where $x_{1}$ and $x_{2}$ are the values of attributes $A_{1}$ and $A_{2}$. And we regard $\mathrm{b}$ as an additional weight, $w_{0}$, We obtain $w_{0}+w_{1} \cdot x_{1}+w_{2} \cdot x_{2}=0$. The weights can be adjusted so that the hyperplanes defining the "sides" of the margin can be written as:

$$
\begin{aligned}
& H 1: w_{0}+w_{1} \cdot x_{1}+w_{2} \cdot x_{2} \geq 1, \forall y_{i}=+1 \\
& H 2: w_{0}+w_{1} \cdot x_{1}+w_{2} \cdot x_{2} \leq-1, \forall y_{i}=-1
\end{aligned}
$$

Combining the two inequalities, we get $y_{i}\left(w_{0}+w_{1} \cdot x_{1}+w_{2}\right.$ $\left.\cdot x_{2}\right) \geq 1 \forall i$. It becomes what is known as a constrained (convex) quadratic optimization problem. Then, after the process, we know the maximal margin is $\frac{2}{\|\mathrm{~W}\|}$, where $\|\mathrm{W}\|$ is the Euclidean norm of $\mathrm{W}$, that is, $\sqrt{(W \cdot W)}$.

\subsection{Logistic Regression}

Logistic regression is a generalized linear regression analysis model. The basic concept of logistic regression is to separate different class in the hyperplane. First, the concept of sigmoid function which is also called logistic function and shown in Eq. (7).

$f(z)=\frac{1}{1+e^{-z}}$, where $\mathrm{z}$ is an independent variable and $\mathrm{e}$ is the natural logarithm base (7).

According to the definition from Kleinbaum et al. (2010), we write $\mathrm{z}$ as a linear sum:

$z=\alpha+\beta_{1} X_{1}+\beta_{2} X_{2}+\ldots+\beta_{k} X_{k}$

where the $X_{i}$ are independent variables and $\alpha$ and the $\beta_{i}$ are constant terms representing unknown parameters $(\forall i=1,2$, ..., k). Based on Eq. (8), we obtain the logistic model in Eq. (9).

$f(z)=\frac{1}{1+e^{\left(-\alpha+\sum \beta_{i} X_{i}\right)}}$ 
Thus, we use maximum likelihood (ML) method to obtain these estimates $\alpha, \beta_{1}, \beta_{2}, \ldots, \beta_{k}$, and then can solve classification problems. MurtiRawat et al. (2020) detected breast cancer using Logistic Regression, K-Nearest Neighbors and Ensemble Learning. Through finding the estimator of $\beta_{i}$, we can get a formula to categorize the $\mathrm{X}$ in the specific class.

\section{Results}

\subsection{Data Collection}

The questionnaire design consisted of three parts: demographic data, perceived risks, and intention to use mobile payment (Yang et al., 2015; Thakur \& Srivastava, 2014). It included 22 questions regarding perceived risks (Table 1): four questions of financial risk, four of privacy risk, four of performance risk, three of psychological risk, four of time risk, and three of security risk. Also, one question for intention to use. We used a five-point Likert scale for all items, as follows: (1) strongly disagree, (2) disagree, (3) neutral, (4) agree, and (5) strongly agree. The question regarding "intention to use mobile payment" will be converted from ordinal to nominal as type of class for classification. Strongly disagree and disagree will be categorized as "low", neutral will be categorized as "medium", and strongly agree and agree will be categorized as "high". The online questionnaire was administered through Google form and two certified translators were used to translate from English to Japanese. Pilot tests (20 participants) were conducted leading to some adjustments in questionnaire items. This research used judgmental sampling method to collect Japanese participants via friends, colleagues as well as families between February and June in 2019 and finally 241 valid participants were collected.

The collected data shows $44 \%$ is male and $56 \%$ is female, while $25.7 \%$ is married and $74.3 \%$ is unmarried. The majority of participants were in the age group of 21 to 30 , which characterized $44.4 \%$ in Japan. The age group of 20 or below is $10.4 \%, 31-40$ is $21.6 \%$, and 41 or above is $23.7 \%$. In education, $66.0 \%$ of Japanese participants had an educational level of bachelor's degree and above and $20.3 \%$ had degree of high school or below. Regarding occupation, students comprised a significant percentage among Japanese respondents (31.5\%), $15.8 \%$ in service industry, $9.5 \%$ in education industry, $7.5 \%$ in sales industry, and $6.2 \%$ in IT industry. $55.5 \%$ of respondents had mobile payment using experience and Apple pay $(30.7 \%)$ and LINE pay $(22 \%)$ are the popular used methods. The popular intensives to use mobile payment include convenience $(58.5 \%)$ and cash back $(22.8 \%)$. Although cashless including credit cards was popular in Japan, there was resistance to mobile payments such as PayPay. This tendency has emerged from a survey conducted by Payments Japan, an organization established by the Ministry of Economy, Trade and Industry (, 2020). The survey showed the willingness to use cashless method increases by age: $52.8 \%$ male and $50.4 \%$ female of $20 \mathrm{~s}, 58.2 \%$ male and $56.2 \%$ female of $30 \mathrm{~s}, 56.4 \%$ male and female of $40 \mathrm{~s}, 54.5 \%$ male and $57 \%$ female of $50 \mathrm{~s}$, and $62.2 \%$ male and $68.4 \%$ female of $60 \mathrm{~s}$. That is, Japanese users showed younger generation may resistant more than older generations toward caseless.

This research conducted one-way-ANOVA analysis for each perceived risk. In Table 1 , security risk $(\mathrm{F}=3.806, p=$ $0.000)$ and privacy risk $(\mathrm{F}=3.566, p=0.000)$ indicates significant difference among all risks. This may reflect the conservative culture of Japan to slow mobile payment usage down. Time risk $(\mathrm{F}=2.688, p=0.001)$ and financial risk $(\mathrm{F}=2.124$, $p=0.008$ ) also show significant difference because Japanese participants' fear of wasting their time trying to use mobile payment and fixing their problems compared to pre-paid cards (e.g., Suica and PASMO). Performance risk $(\mathrm{F}=1.33, p=$ $0.191)$ and psychological risk $(\mathrm{F}=1.788, p=0.051)$ present insignificant difference among all risks. We infer that information and communication technology industry has become mature in Japan and people trust the used technologies including mobile payment method.

\subsection{Metrics}

We conducted 10-fold cross-validation for decision trees learning algorithm. Decision trees are built using training and testing data with the outcomes generating the confusion matrix. This confusion matrix is used to measure the performance of the built model regarding predicted case and true class. Different evaluation measures are used such as accuracy, precision, recall, and F1-score. Accuracy is the ratio of number of correct predictions to the total number of input samples (Eq.(10)). Precision is the number of true positives divided by the total number of respondents labelled as belonging in the positive class (Eq.(11)). Recall is the number of true positives divided by the total number of respondents who actually belong in the positive class (Eq.(12)). Finally, $F_{1}$-score is a harmonic measure calculated by weighted precision and recall (Eq.(13)). In particular, TP (true positive) indicates the number of respondents correctly labelled as belonging in the positive class. $F N$ (false negative) wrongly denotes that a predicted class does not exist, when it does, while FP (false positive) wrongly indicates that a predicted class exists, when it does not.

$$
\begin{aligned}
& \text { Accuracy }=\frac{T P+T N}{T P+T N+F P+F N} \\
& \text { Precision }=\frac{T P}{T P+F P} \\
& \text { Recall }=\frac{T P}{T P+F N}
\end{aligned}
$$


Table 1 Summary of differences in perceived risks

*Number of Respondents: TW: 242; CN: 243; JP: 241

1. The use of mobile payment (m-payment) would cause the exposure of personal bank accounts and passwords.

2. Malicious or unreasonable charging could occur.

3. A careless operation could lead to a surprising loss.

4. The use of m-payment could cause financial risk.

Privacy Risk

5. Private information could be misused, inappropriately shared, or sold.

6. Personal information could be intercepted or accessed.

7. Payment information could be collected, tracked, and analysed.

8. Privacy could be exposed when using m-payment.

\section{Performance Risk}

9. The payment system might be unstable or blocked.

10. The payment system does not work as expected.

11. The performance level might be lower than designed.

12. The service performance might not match its advertised level.

Psychological Risk

13. Mobile payment would cause unnecessary tension (e.g., concerns about errors).

14. A system malfunction in m-payment could cause unwanted anxiety and confusion.

15. The usage of m-payment could cause discomfort.

\section{Time Risk}

16. Time loss could be caused by instability and low speed.

17. It might take too much time to learn how to use mobile payment.

18. More time is required to fix payment errors offline.

19. Using m-payment may waste time.

\section{Security Risk}

20. There might be mistakes, since the accuracy of the inputted information is difficult to check from the screen.

21. The battery of the mobile phone might run out or the connection could be interrupted while paying.

22. The bill information might be typed wrongly.

\begin{tabular}{|c|c|c|}
\hline Source & Mean & $\mathrm{F} / p$ \\
\hline \multirow[t]{24}{*}{ Yang et al. (2015) } & 2.51 & $\begin{array}{r}\mathrm{F}=2.124 / \\
p=0.008\end{array}$ \\
\hline & 2.56 & \\
\hline & 2.20 & \\
\hline & 2.73 & \\
\hline & 2.55 & \\
\hline & 2.84 & $\begin{array}{c}\mathrm{F}=3.566 / \\
p=0.000\end{array}$ \\
\hline & 2.67 & \\
\hline & 2.80 & \\
\hline & 3.11 & \\
\hline & 2.77 & \\
\hline & 2.56 & $\begin{array}{c}\mathrm{F}=1.33 / \\
p=0.191\end{array}$ \\
\hline & 2.99 & \\
\hline & 2.31 & \\
\hline & 2.41 & \\
\hline & 2.55 & \\
\hline & 2.82 & $\begin{array}{r}\mathrm{F}=1.788 / \\
p=0.051\end{array}$ \\
\hline & 2.61 & \\
\hline & 3.13 & \\
\hline & 2.71 & \\
\hline & 2.76 & $\begin{array}{c}\mathrm{F}=2.688 / \\
p=0.001\end{array}$ \\
\hline & 2.98 & \\
\hline & 2.76 & \\
\hline & 3.07 & \\
\hline & 2.20 & \\
\hline \multirow[t]{4}{*}{$\begin{array}{l}\text { Thakur and Srivastava } \\
\text { (2014) }\end{array}$} & 2.92 & $\begin{array}{c}\mathrm{F}=3.806 / \\
p=0.000\end{array}$ \\
\hline & 2.70 & \\
\hline & 3.34 & \\
\hline & 2.74 & \\
\hline
\end{tabular}

$$
F_{1}-\text { Score }=\frac{2 * \text { Precision } * \text { Recall }}{\text { Precision }+ \text { Recall }}
$$

\subsubsection{Analysis of Decision Trees}

The generated binary tree contains 91 nodes and 46 leaves (Fig. 3). The setting for tree pruning includes at least two instances in leaves, at least five instances in internal nodes, and maximum depth is 100 . The tree stops splitting when majority reaches $95 \%$. This research selected the coverage rate of data that is higher than $60 \%$ for the generated rules of three classes. The results of low intention indicated the rule: Q23 (are you satisfied with existing mobile payment method) is lower and equal than 2 and Q2 (malicious or unreasonable charging could occur) is lower and equal than 3 . Users from category of low intention had low satisfaction of mobile payment and less concerned about malicious charging of mobile payment, which refers to financial risk. That is, less concern about unexpected monetary loss of mobile payment is the feature of category of low intention.

The results of medium intention can be classified into two rules. First, Q23 (are you satisfied with existing mobile payment method) is higher than 2 and lower and equal than 3 , and Q16 (time loss could be caused by instability and low speed) is lower and equal than 3. First type of users from category of 


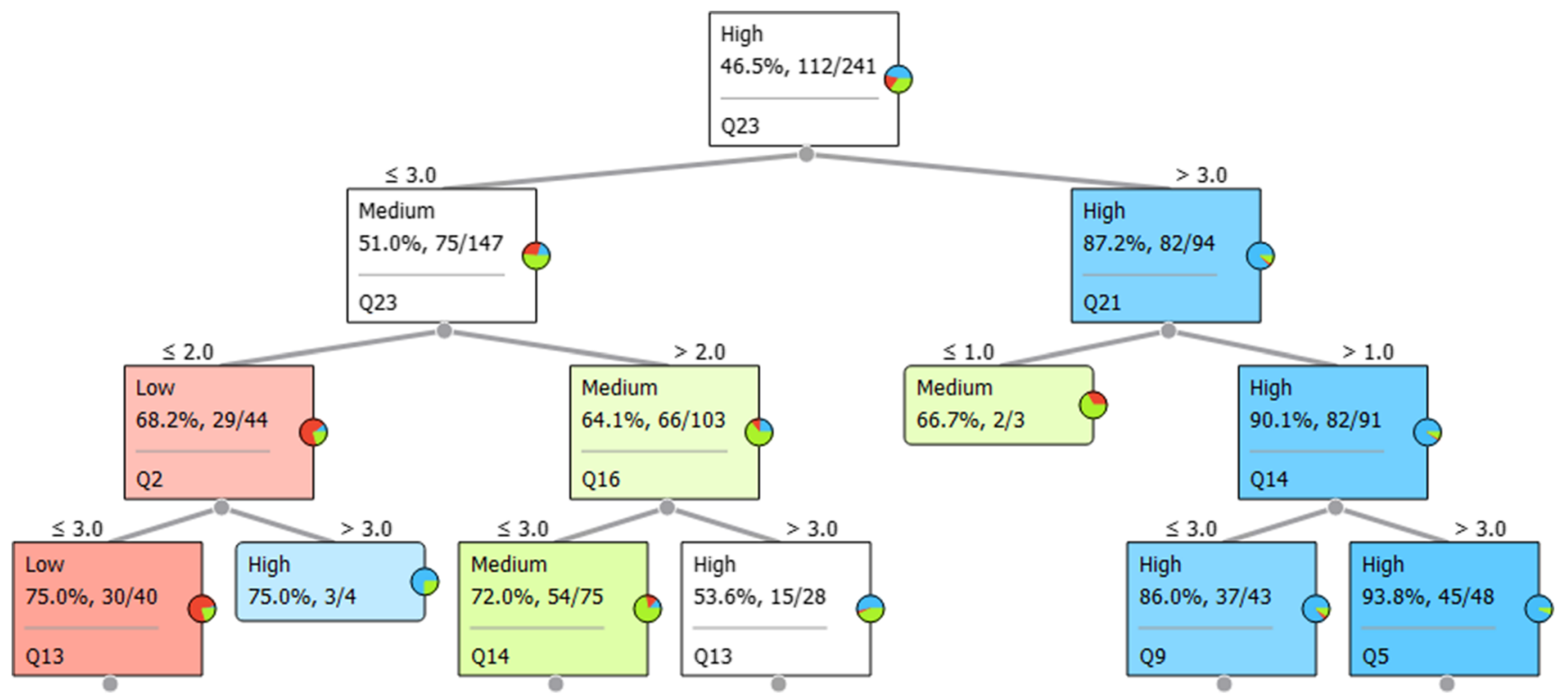

Fig. 3 A generated decision tree

medium intention had medium-low satisfaction of mobile payment and less concern about time risk. Second, Q23 (are you satisfied with existing mobile payment method) is higher than 3 and Q21 (the battery of the mobile phone might run out or the connection could be interrupted while paying) is lower and equal to 1 . Second type of users from category of medium intention had higher satisfaction and less concern about the disconnection of mobile phone when paying, which refers to security risk. Hence, user from medium intention had two features: (1) medium-low satisfaction and less concerned about time risk and (2) medium-high satisfaction and less concern about security risk. The outcomes of high intention generated the rule: Q23 (are you satisfied with existing mobile payment method) is higher than 3, Q21 (the battery of the mobile phone might run out or the connection could be interrupted while paying) is higher than 1, and Q14 is lower and equal to 3 (a system malfunction in m-payment could cause unwanted anxiety and confusion). Users from the category of high intention had higher satisfaction, less concern about security risk, and less concern about psychological risk.

\subsection{Model Comparison}

This research applied methods of decision tree, kNN, Naïve Bayes, SVM, and Logistic Regression to validate the models in terms of accuracy, prediction, recall, F1-score (Fig. 4). Decision trees shows highest accuracy $(0.668)$, precision (0.665), recall (0.668), and F1-score (0.666) among five models. SVM has the second highest accuracy $(0.66)$, precision (0.663), recall (0.66), and F1-score (0.644) among kNN, Naive Bayes, Logistic Regression, and SVM. The area under ROC was also estimated which reveals all models have similar discrimination. The value of area under ROC (AUC) for decision trees is $0.738, \mathrm{kNN}$ is 0.749 , Naïve Bayes is 0.758 , SVM is 0.844 , and logistic regression is 0.776 as shown in Fig. 5, Fig. 6, and Fig. 7. Compared with all indicators, this study showed decision trees shows the optimal outcomes of classification among all models.

\subsection{Discussion}

This research summarizes the results for each age group as show in Table 2. The age between 21 and 40 showed more mobile payment using experience who belong to young and major shoppers of samples. Using experience showed top reason to use mobile payment for all age groups was convenience and second reason was the incentive of cash back from banks. The results of ANOVA analysis indicate security risk presents significant difference for the age group 20 or below $(\mathrm{F}=9.02$, $p=0.000)$ and 41 or above $(\mathrm{F}=2.021, p=0.045)$. This means young and old age groups may concern about data security problem on mobile payment. Time risk is significant for the age group between 31 and $40(\mathrm{~F}=2.667, p=0.012)$ and 41 or above $(\mathrm{F}=2.22, p=0.023)$. We infer that losing or wasting time via mobile payment may cause anxiety when Japanese people have time pressure on job and family. Privacy risk is significant for the age group $21-30(\mathrm{~F}=1.998, p=0.021)$ and 41 or above $(\mathrm{F}=2.459, p=0.011)$. This may because major shoppers used mobile payment more often and privacy is the crucial concern for using mobile payment.

Despite the mobile transaction is popular, cash is still the most preferred payment in Japan, accounting for $80 \%$ of all transactions during 2018 (Fawthrop, 2019). It is believed that the habit to pay in Japan is still cash dominant even credit card is popular and mobile payment is raising. Changing the habit is more difficult than understanding it. The theory of risk and 
Fig. 4 Model comparison of classification outcomes

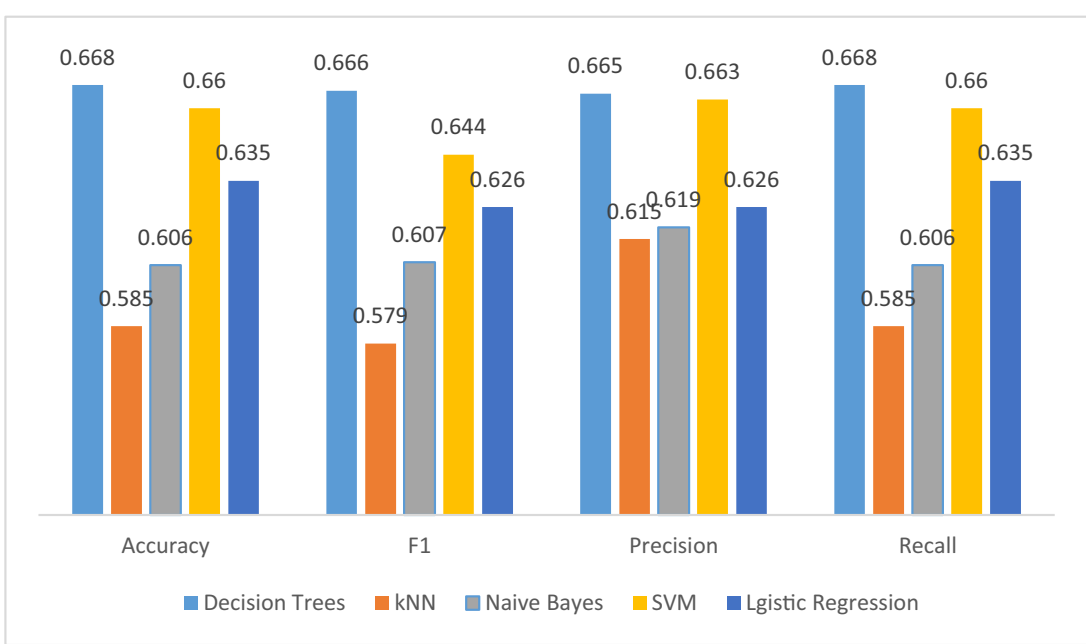

attractiveness (Weber et al., 1992) can help us understand the importance of risk, which indicated that risk judgements are more sensitive to losses and zero outcomes, while attractiveness judgements are more sensitive to gains. The theory explains people are conservative when facing the risk of losses rather than attractiveness of gains. According to Hakuhodo's consumer research in $2017,51 \%$ of Japanese people against cashless society. $20 \%$ of Japanese people are concerned with spending too much via credit card that researchers also confirmed may increase willingness-to-pay (Prelec \& Simester, 2001). Most importantly, Japanese people are worried about security and data breach of cashless payment methods.
Our findings showed effects of perceived risks on intention to use mobile payment of Japanese users (Table 3). We also assume degree of intention to use mobile payment is consistent with satisfaction of usage. Financial risk shows effects on low intention to use mobile payment. The average score of financial risk were the lowest to all age groups. We infer that infrastructure of information and communication technology is mature in Japan that may cause low chance of unreasonable charging. Privacy and performance risks also influence low intention to use mobile payment. The average score of two risks were important to two age groups between 21 and 40 . That is, safe, secured, reliable, and fast mobile payment

Fig. 5 ROC of target class of high

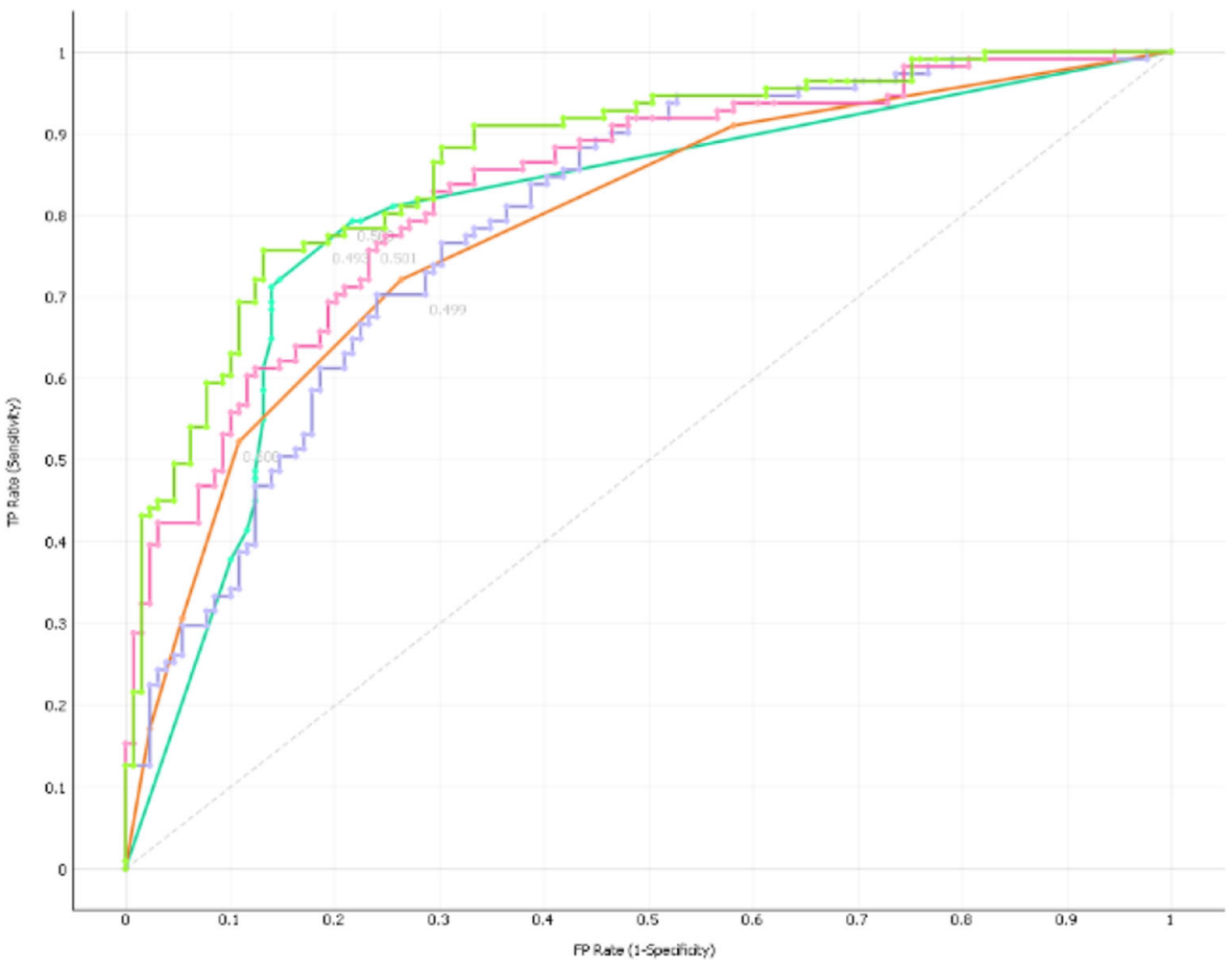


Fig. 6 ROC of target class of medium

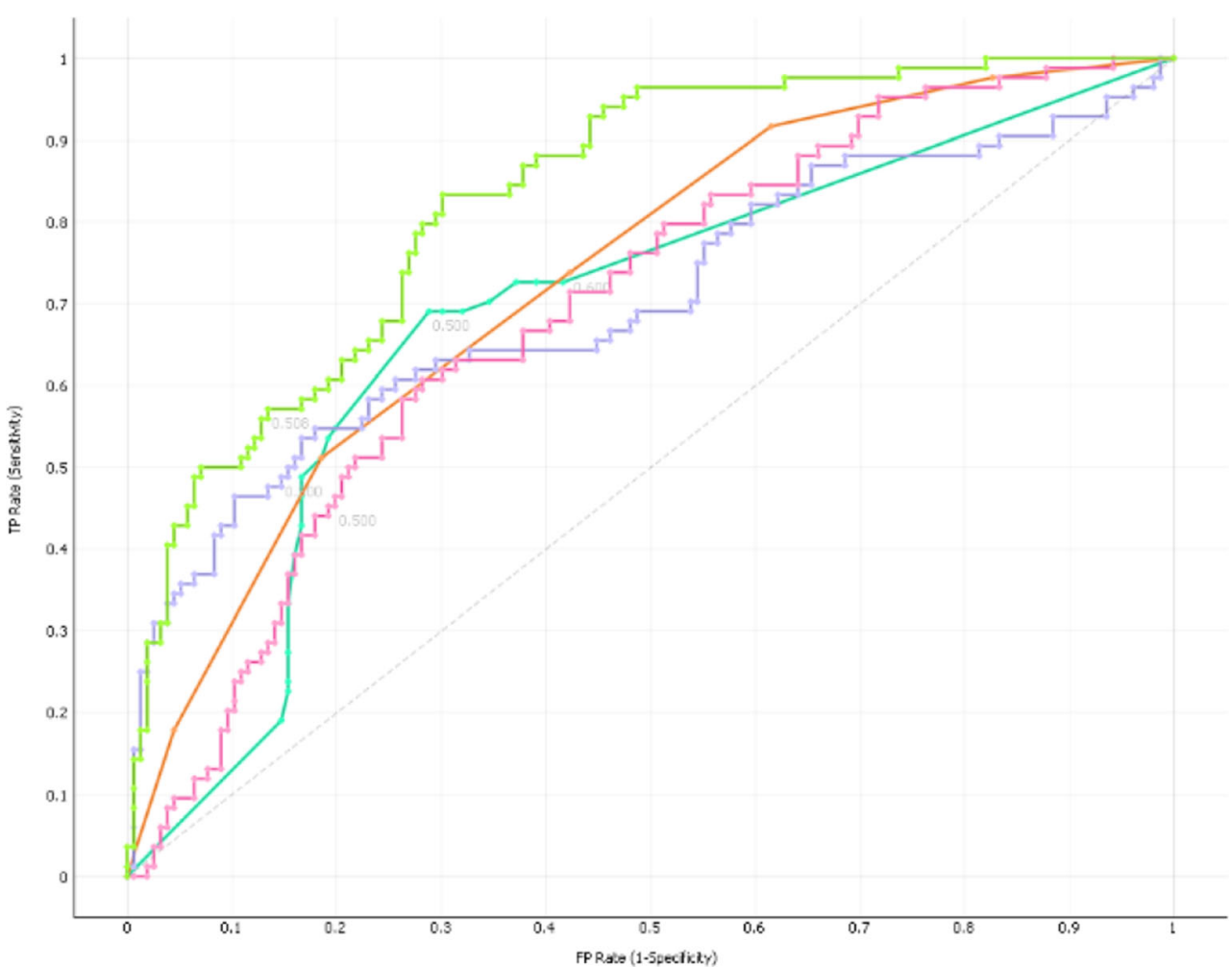

environment are more important the category of low intention. In addition to privacy and performance risks, psychological and security risks show effect on high intention to use mobile payment. The reliability of mobile device (e.g., battery and function), financial loss, safe, secured, reliable, and fast mobile payment environment are key drivers to users. The average scores of security risk were the highest for all age groups and psychological risk shows second highest average scores

Fig. 7 ROC of target class of low

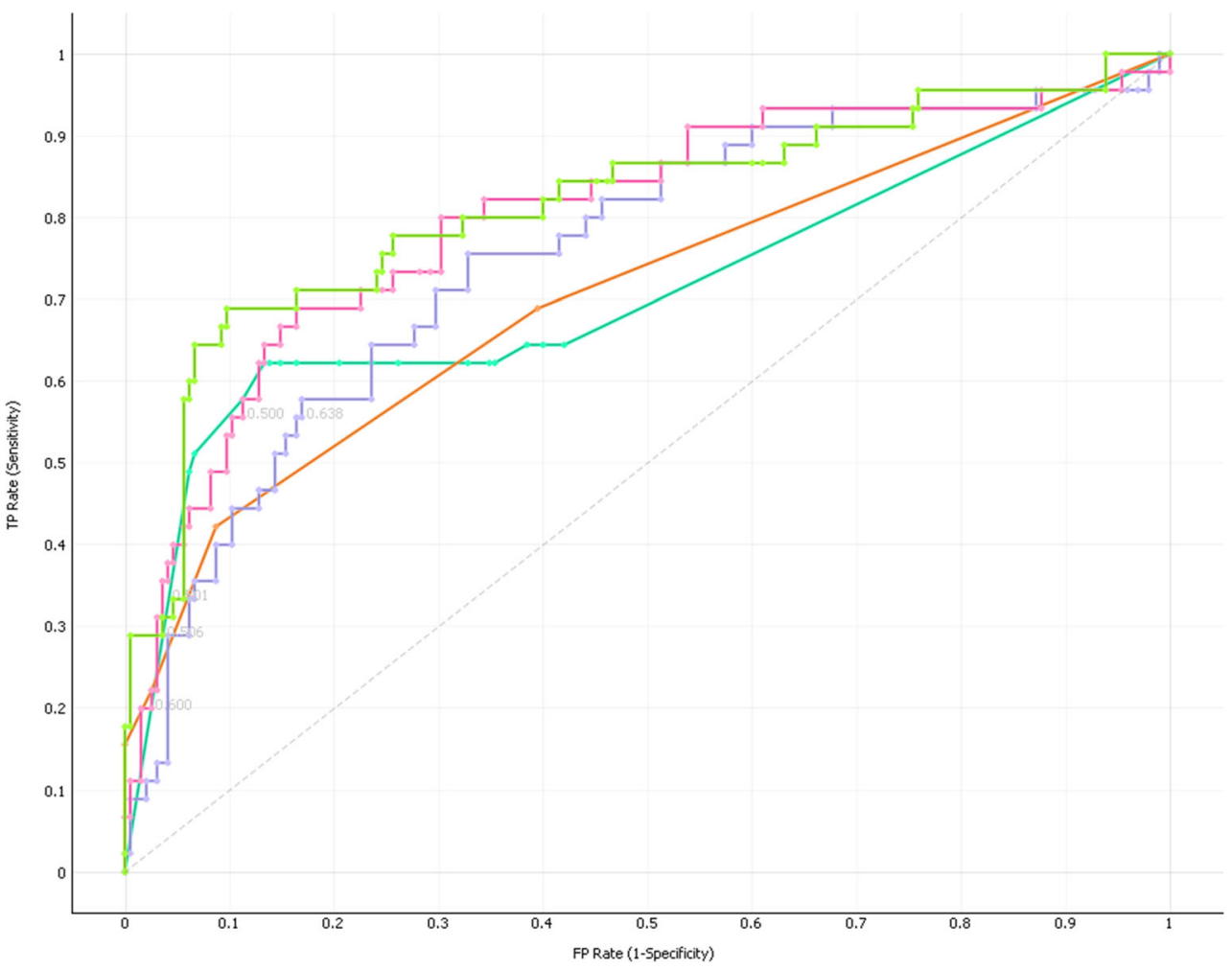




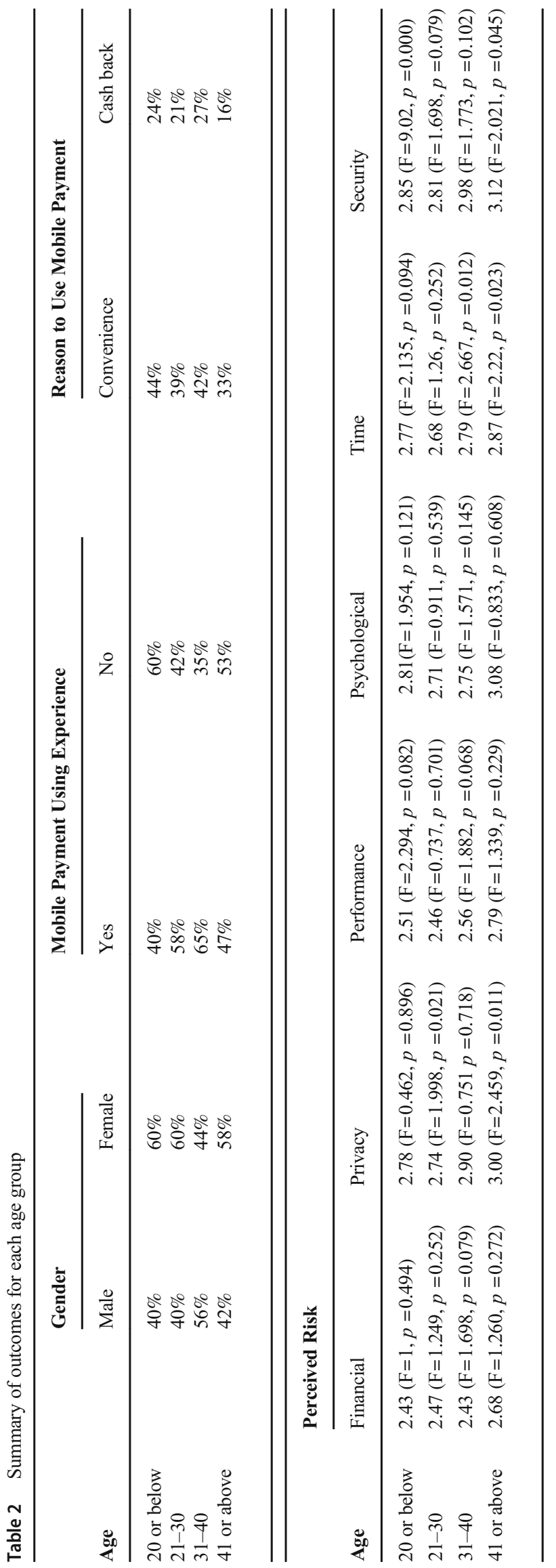

for the age group of 20 or below and 41 or above. Particularly, the reliable mobile payment platform may derive acceptable performance risk for the category of high intention.

Moreover, medium intention to use mobile payment can be categorized into medium-low satisfaction and medium-high satisfaction. Specifically, time risk shows effect on mediumlow satisfaction because users expect quick mobile payment process. The average scores of time risk were also fairy high to all age groups. Security risk shows effect on medium-high satisfaction because users may trust the reliability of mobile device and expect the mobile payment process is trustworthy. Monetary loss, safe, reliable, and fast mobile payment environment are also important to the category of medium-high satisfaction. Most importantly, our analysis indicated privacy and performance risks show effects on all categories. Japanese users concerned about safe and reliability of mobile payment methods as the top priority. By showing the effects of all perceived risks on intention to use mobile payment, firms can emphasize on specific risk to handle and promote the usage for different age groups. Our results are also in accordance with existing reports, which specified $70 \%$ of population in Japan still preferred cash, worried about security issue, expected secured and easy process, and were afraid of tracking non-cash cashless methods.

\section{Implications}

This research attempts to discover the perceived risk of mobile payment from Japanese users. We classified users into low, medium, and high intention to use mobile payment methods. The findings also showed that monetary loss is not important to low intention users, time and security risks are not influential to medium intention users, and security and psychological risks are not crucial to high intention users. That is, privacy and performance are the joint important perceived risk to all users, which can match to the cultural background in Japan (e.g., Japanese people are more conservative toward mobile payment). Companies should emphasize on data protection and functions of mobile payment for further improvement. For low intention users, companies may improve secured, safe, and fast mobile payment process. For medium intention users, firms should avoid monetary loss and comfortable mobile payment environment. For high intention users, monetary loss, and fast mobile payment process are critical. Improving ease of use and usefulness of mobile payment can relief the tension of perceived risk. In Japanese culture, companies need to step-by-step unlock the tension of perceived risk that may enhance the use of mobile payment incrementally. When the users accept the risk and are willing to use mobile payment methods, the profits will be increased for enterprises simultaneously. 
Table 3 Effects of perceived risks and categories of Japanese users

Financial Risk Privacy Risk Performance Risk Psychological Risk Time Risk Security Risk

Low intention (Low Satisfaction)

Medium intention (Medium-low Satisfaction)

(Medium-high Satisfaction)

High intention (High Satisfaction)

$\begin{array}{ll}++ & ++ \\ ++ & ++ \\ ++ & ++ \\ ++ & ++\end{array}$

$++$

$+$

$+$

$+$

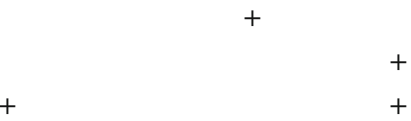

+ means less than half of variables influence on intention to use mobile payment

++ means more than half of variables influence on intention to use mobile payment

The wave of $5 \mathrm{G}$ leads the development of mobile applications and services. In Japan, mobile payment methods have been promoted and applied widely because of Tokyo 2020 Olympics. Japan is one of the countries that cash is still the major payment method nowadays. The 2020 McKinsey Global Payment Report also indicated that estimated 54\% of transactions was cash in Japan (top 1 country) in the mature market, following by Singapore (39\%). The evidence presents while the mobile payment usage is not low but people still prefer cash usage in Japan. The spread of pandemic (Covid19) may also impact all stakeholders of mobile payment (e.g., government, companies, and users) and push digital transformation on cashless society. Our findings expect to contribute to the countries with high percentage of cash used in transactions in the emerging market (e.g., Indonesia, Brazil, and Argentina) and cash-loyal countries (e.g., Germany and Italy) according to BCG survey in 2020.

\section{Conclusion and Limitations}

The wave of 5G increases the opportunities of mobile applications including mobile payment. While the infrastructure has become mature, companies change the focus to mobile payment behavior. In Japan, cashless is not yet popular but government and companies are devoted to the development of mobile payment methods. In 2020, the mobile payment methods are considered to be widely applied and used because of Tokyo 2020 Olympics. Hence, understanding the perceived risks on mobile payment from Japanese users are critical. This research collected 241 Japanese users and applied decision trees algorithm. Six types of perceived risks (financial, privacy, performance, psychological, security, and time) were used and the categorized class is intention to use mobile payment (low, medium, and high). We also compared different competitive models to examine the performance, including decision trees, kNN, SVM, logistic regression, and Naïve Bayes. The outcomes showed decision trees outperformed among all models in terms of accuracy, precision, recall, and F1measure.

By analyzing the generated decision trees, the findings indicated that safe, secured, reliable, and fast mobile payment environment are more important to low intention users, which means less concerns about financial risk. Financial loss, safe, secured, reliable, and fast mobile payment environment are more important to medium intention users, which means less concerns about time and security risk. Monetary loss, reliable, and fast mobile payment environment are more important to high intention users, which means less concerns about security risk \& psychological risk. Furthermore, two limitations are identified in this study. First, more samples can be extended to generalize the discovered outcomes. Japanese users are conservative but their perceptions are valuable for the development of mobile payment. Second, the different viewpoints can be used to supplement our study which merely focus on perceived risks. Although risk is more influential than attractiveness, the perceived gains can help companies balance the influence of adoption of mobile payment The spread of corona infection has led to the increasing prevalence of mobile payments in Japanese society. How people's concerns about perceived risks are changing in the post-corona era should also continue to be assessed.

\section{References}

Asia Pacific eCommerce and Payments Guide 2020 (2020). Available at: https://cdn2.hubspot.net/hubfs/5246303/Asia\%20Pacific\% 20eCommerce\%20and\%20Payments\%20Guide\%202020.pdf

GSMA. (2019). The mobile economy Asia Pacific 2019 report. GSM Associates. https://www.gsmaintelligence.com/research/?file $=$ fe 8735424e3058f98c3a83bc57bc2af5\&download.

Hakuhodo's Consumer Research (2017). Available at: https://www. hakuhodo.co.jp/uploads/2017/12/20171215.pdf

The Cashless Promotion Council Payments Japan (2020). https://www. paymentsjapan.or.jp/ (in Japanese).

The Cashless Promotion Council Payments Japan Consumer and Business Insight Survey (2020). Available at: https://www. paymentsjapan.or.jp/wordpress/wp-content/uploads/2021/05/ insights_fy2020.pdf (in Japanese).

The 2020 McKinsey Global Payment Report (2020). Available at: https:// www.mckinsey.com/ /media/mckinsey/industries/financial\% 20 services/our $\% 20 \mathrm{insights} /$ accelerating $\% 20$ winds $\% 20$ of $\%$ 20change $\% 20$ in $\% 20$ global $\% 20$ payments $/ 2020$-mckinseyglobalpayments-report-vf.pdf 
Payments Japan Consumer Insight Survey 2020, Available at: https:// www.paymentsjapan.or.jp/wordpress/wp-content/uploads/2021/05/ insights fy2020.pdf.

Agrawal, R., Ghosh, S., Imielinski, T., Iyer, B., \& Swami, A. N. (1992, August). An interval classifier for database mining applications. In $V L D B$ (Vol. 92, pp. 560-573).

Albashrawi, M., \& Motiwalla, L. (2019). Privacy and personalization in continued usage intention of mobile banking: An integrative perspective. Information Systems Frontiers, 21(5), 1031-1043.

Arvidsson, N. (2014). Consumer attitudes on mobile payment servicesresults from a proof of concept test. International Journal of Bank Marketing, 32(2), 150-170.

Baganzi, R., \& Lau, A. K. (2017). Examining trust and risk in mobile money acceptance in Uganda. Sustainability, 9(12), 22-33.

Breiman, L., Friedman, J., Stone, C. J., \& Olshen, R. A. (1984). Classification and regression trees. CRC Press.

Cao, X., Yu, L., Liu, Z., Gong, M., \& Adeel, L. (2018). Understanding mobile payment users' continuance intention: A trust transfer perspective. Internet Research, 28(2), 456-476.

Chen, X., \& Li, S. (2017). Understanding continuance intention of mobile payment services: An empirical study. Journal of Computer Information Systems, 57(4), 287-298.

Choi, Y., \& Choi, H. (2017). Risk factors affecting trust and satisfaction in mobile payment systems, International Information Institute (Tokyo). Information, 20(8A), 5527-5532.

Cocosila, M., \& Trabelsi, H. (2016). An integrated value-risk investigation of contactless Mobile payments adoption. Electronic Commerce Research and Applications, 20, 159-170.

Dahlberg, T., Mallat, N., Ondrus, J., \& Zmijewska, A. (2008). Past, present and future of mobile payments research: A literature review. Electronic Commerce Research and Applications, 7(2), 165-181.

De, L., Liébana-Cabanillas, I. R. F., Sánchez-Fernández, J., \& MuñozLeiva, F. (2018). Mobile payment is not all the same: The adoption of mobile payment systems depending on the technology applied. Change (In press).

Dey, P. K. (2002). Project risk management: A combined analytic hierarchy process and decision tree approach. Cost Engineering, 44(3), 13-27.

Dighe, S. (2018) Global mobile payments industry is expected to grow at a CAGR of $33.8 \%$ fom 2017 to 2023. AMR, Business Indiser. Available at: https://markets.businessinsider.com/news/stocks/ global-mobile-payments-industry-is-expected-to-grow-at-a-cagr-of33-8-from-2017-to-2023-amr-1027500286.

Dinh, V. S., Nguyen, H. V., \& Nguyen, T. N. (2018). Cash or cashless? Promoting consumers' adoption of mobile payments in an emerging economy. Strategic Direction, 34(1), 1-4.

Fahey, R. (2019) Cashless payments struggle in cash-loving Japan, 2019, https://www.tokyoreview.net/2019/01/cashless-payments-japan/

Fawthrop, A. (2019) Adoption of Mobile Payments and Digital Wallets Growing in Japan. Available at: https://www.nsbanking.com/news/ mobile-payments-japan/

Featherman, M. S., \& Pavlou, P. A. (2003). Predicting e-services adoption: A perceived risk facets perspective. International Journal of Human-Computer Studies, 59(4), 451-474.

Flavián, C., Guinalíu, M., \& Gurrea, R. (2006). The role played by perceived usability, satisfaction and consumer trust on website loyalty. Information \& Management, 43(1), 1-14.

Forsythe, S. M., \& Shi, B. (2003). Consumer patronage and risk perceptions in internet shopping. Journal of Business Research, 56(11), 867-875.

Gillett, P. L. (1976). In-home shoppers: An overview. The Journal of Marketing, 40(4), 81-88.

Han, J., Pei, J., \& Kamber, M. (2012). Data mining: Concepts and techniques. Elsevier.

Hayashi, F., \& Bradford, T. (2014). Mobile payments: merchants' perspectives. Economic Review, 99(1), 5-30.
Kabari, L. G., \& Nwachukwu, E. O. (2013). Credit risk evaluating system using decision tree-neuro based model. International Journal of Engineering Research \&Technology, 2(6), 2738-2745.

Khalilzadeh, J., Ozturk, A. B., \& Bilgihan, A. (2017). Security-related factors in extended UTAUT model for NFC based mobile payment in the restaurant industry. Computers in Human Behavior, 70(4), 460-474.

Kim, J., \& Lennon, S. J. (2013). Effects of reputation and website quality on online consumers' emotion, perceived risk and purchase intention: Based on the stimulus-organism-response model. Journal of Research in Interactive Marketing, 7(1), 33-56.

Kleinbaum, D. G., \& Klein, M. (2010). Introduction to logistic regression. In Logistic regression (pp. 1-39). Springer.

Kolsaker, A., \& Payne, C. (2002). Engendering trust in e-commerce: A study of gender-based concerns. Marketing Intelligence \& Planning, 20(4), 206-214

Liébana-Cabanillas, F., \& Lara-Rubio, J. (2017). Predictive and explanatory modeling regarding adoption of mobile payment systems. Technological Forecasting and Social Change, 120, 32-40.

Liébana-Cabanillas, F., Molinillo, S., \& Ruiz-Montañez, M. (2019). To use or not to use, that is the question: Analysis of the determining factors for using NFC mobile payment systems in public transportation. Technological Forecasting and Social Change, 139, 266276.

Lim, N. (2003). Consumers' perceived risk: Sources versus consequences. Electronic Commerce Research and Applications, 2(3), 216-228.

Luarn, P., \& Lin, H. H. (2005). Toward an understanding of the behavioral intention to use mobile banking. Computers in Human Behavior, 21(6), 873-891.

Liu, J., Kauffman, R. J., \& Ma, D. (2015). Competition, cooperation, and regulation: Understanding the evolution of the mobile payments technology ecosystem. Electronic Commerce Research and Applications, 14(5), 372-391.

Mann, B. J. S., \& Sahni, S. K. (2013). Role of trust and customer loyalty in reducing perceived security risk in internet banking. International Journal of Electronic Business, 10(4), 331-354.

MarketsandMarkets (2019). Digital payments market by component, deployment type, organization size, vertical, and region - global forecast to 2025. Available at: https://www.marketsandmarkets.com/ Market-Reports/digital-payment-market-209834053.html.

METI, Ministry of Economy, Trade and Industry report "current status and significance of cashless" (2020), https://www.meti.go.jp/policy/ mono info service/cashless/image pdf movie/about cashless.pdf (in Japanese).

Mordor Intelligence (2018). Cyber (liability) insurance market - AsiaPacific trends, industry competitiveness, disruptions \& innovations, forecasts to 2022. Available at: https://www.mordorintelligence. com/industry-reports/asia-pacific-cyberliability-insurance-market.

MurtiRawat, R., Panchal, S., Singh, V. K., \& Panchal, Y. (2020). Breast Cancer detection using K-nearest neighbors, logistic regression and ensemble learning. In 2020 international conference on electronics and sustainable communication systems (ICESC) (pp. 534-540). IEEE.

Nel, J., Heyns, N., (2017) The in-store factors influencing the use intention of proximity mobile-payment applications, Management Dynamics: Journal of the Southern African Institute for Management Scientists, 26(2), 2-20.

Oliveira, T., Thomas, M., Baptista, G., \& Campos, F. (2016). Mobile payment: Understanding the determinants of customer adoption and intention to recommend the technology. Computers in Human Behavior, 61, 404-414.

Park, J., Amendah, E., Lee, Y., \& Hyun, H. (2019). M-payment service: Interplay of perceived risk, benefit, and trust in service adoption. Human Factors and Ergonomics in Manufacturing \& Service Industries, 29(1), 31-43. 
Prelec, D., \& Simester, D. (2001). Always leave home without it: A further investigation of the credit-card effect on willingness to pay. Marketing Letters, 12(1), 5-12.

Qasim, H., \& Abu-Shanab, E. (2016). Drivers of mobile payment acceptance: The impact of network externalities. Information Systems Frontiers, 18(5), 1021-1034.

Quinlan, J.R. (1993) C4.5: Programs for machine learning, Morgan Kaufmann publishers, New York, 1993.

Ramezankhani, A., Kabir, A., Pournik, O., Azizi, F., \& Hadaegh, F. (2016). Classification-based data mining for identification of risk patterns associated with hypertension in middle eastern population: A 12-year longitudinal study. Medicine, 95(35), 325-335.

Saridakis, G., Benson, V., Ezingeard, J. N., \& Tennakoon, H. (2016). Individual information security, user behaviour and cyber victimisation: An empirical study of social networking users. Technology Forecasting and Social Change., 102, 320-330.

Shao, Z., Zhang, L., Li, X., \& Guo, Y. (2019). Antecedents of trust and continuance intention in mobile payment platforms: The moderating effect of gender. Electronic Commerce Research and Applications, 33(1), 27-56.

Thakur, R., \& Srivastava, M. (2014). Adoption readiness, personal innovativeness, perceived risk and usage intention across customer groups for mobile payment services in India. Internet Research, 24(3), 369-392.

Trachuk, A., \& Linder, N. (2017). The adoption of mobile payment services by consumers: an empirical analysis results. Business and Economic Horizons (BEH), 13(3), 383-408.

Weber, E. U., Anderson, C. J., \& Birnbaum, M. H. (1992). A theory of perceived risk and attractiveness. Organizational Behavior and Human Decision Processes, 52(3), 492-523.

Xin, H., Techatassanasoontorn, A. A., \& Tan, F. B. (2015). Antecedents of consumer trust in mobile payment adoption. Journal of Computer Information Systems, 55(4), 1-10.

Yang, Y., Liu, Y., Li, H., \& Yu, B. (2015). Understanding perceived risks in mobile payment acceptance. Industrial Management \& Data Systems, 115(2), 253-269.

Zhou, T. (2013). An empirical examination of continuance intention of mobile payment services. Decision Support Systems, 54, 10851091.

Zhou, T. (2014). Understanding the determinants of mobile payment continuance usage. Industrial Management \& Data Systems, 114(6), 936-948.

Zhu, D. H., Lan, L. Y., \& Chang, Y. P. (2017). Understanding the intention to continue use of a mobile payment provider: An examination of alipay wallet in China. International Journal of Business \& Information, 12(4), 369-390.
Publisher's Note Springer Nature remains neutral with regard to jurisdictional claims in published maps and institutional affiliations.

Wei-Lun Chang is the Associate Professor of Department of Business Management in National Taipei University of Technology. He completed his Ph. D. degree at Department of Management Information Systems at National Chengchi University. His research areas include social media, sentiment, and technology in education. His work has appeared in Communications of the ACM, Information Systems Frontiers, Internet Research, Electronic Commerce Research and Applications, KnowledgeBased Systems, European Journal and Operational Research, Transportation Research Part E: Logistics and Transportation Review, and Journal of Information Science.

Li-Ming Chen is an Associate Professor in the Department of Business Administration at National Chengchi University, Taiwan. He received his MS in Industrial and Operations Engineering at the University of Michigan, USA, and his PhD in Industrial and Systems Engineering from University of Florida, USA. His research interests include supply chain management and the interface between operations and information. His work has appeared in academic journals such as European Journal and Operational Research, Naval Research Logistics, Transportation Research Part E: Logistics and Transportation Review, and Journal of the Operational Research society.

Takako Hashimoto graduated from the Ochanomizu University in Japan, and received a Ph.D. in computer science, specialization in multimedia information processing, from the Graduate School of Systems and Information Engineering of University of Tsukuba in 2005. She worked at the software R\&D center of Ricoh Co. Ltd., in Japan for 24 years, and participated in the development of many software products as a technical leader. From April of 2009, she was involved in Chiba University of Commerce as Associate Professor. In 2015, she became Professor of Chiba University of Commerce. In 2015, she stayed at University of California, Los Angeles as a visiting researcher. In 2016, she has become the Vice President of Chiba University of Commerce in 2018. She is served as a Board Member of the Database Society of Japan and Chair of IEEE Japan Council. Fellow of the Information Processing Society of Japan. She has focused on the data mining research and the social media analysis, especially topic extraction from millions of tweets related to disasters and topical problems. In 2019, she received the IEEE 2019 MGA Larry K. Wilson Transnational Award, and Fellow of the Information Processing Society of Japan. 